PROCEEDINGS OF THE

AMERICAN MATHEMATICAL SOCIETY

Volume 131, Number 2, Pages 409-414

S 0002-9939(02)06476-6

Article electronically published on September 17, 2002

\title{
ON THE NUMBER OF ZEROS OF CERTAIN HARMONIC POLYNOMIALS
}

\author{
DMITRY KHAVINSON AND GRZEGORZ ŚWIA̧TEK
}

(Communicated by Juha M. Heinonen)

\begin{abstract}
Using techinques of complex dynamics we prove the conjecture of Sheil-Small and Wilmshurst that the harmonic polynomial $z-\overline{p(z)}, \operatorname{deg} p=$ $n>1$, has at most $3 n-2$ complex zeros.
\end{abstract}

\section{INTRODUCTION}

Let $h(z):=p(z)-\overline{q(z)}$ be a harmonic polynomial of degree $n>1$ where $p, q$ are analytic polynomials of degree $n$ and $m, m<n$. The following question was raised by T. Sheil-Small [6: what is the upper bound on the number of zeros of $h$ ? $\mathrm{He}$ conjectured that the sharp upper bound was $n^{2}$. His former student A. Wilmshurst has proved this in his thesis [7] by demonstrating the upper bound using Bézout's theorem and also showing by examples that for $m=n, n-1$ that bound was sharp. Some of Wilmshurst's results were independently discovered by Bshouty et al. [1]. However, for $m<n-1$ it was suggested in [7] that the upper bound should be much lower, in particular Wilmshurst conjectured that for $m=1$ the number of zeros of $h(z)$ does not exceed $3 n-2$. The purpose of this note is to prove this result by using certain well-known techniques from complex dynamics. This is not at all surprising since in that case the zeros of $h$ could be thought of as finite fixed points of the mapping $z \rightarrow \overline{p(z)}$ of the Riemann sphere. It must be mentioned that the first author's attention to the problem was drawn by a question posed by D. Sarason, who jointly with B. Crofoot proved in [5] the $3 n-2$ conjecture for $n=3$ (it is trivial for $n=2$ ). Also, Crofoot and Sarason obtained in [5] several intriguing reformulations of the problem in terms of coercive estimates of some linear operators on finite-dimensional spaces.

The authors are greatly indebted to D. Sarason for valuable discussions of the problem, relevant references and for sharing his and Crofoot's unpublished work with us. We also thank the referee for a quick report and useful comments.

\section{Preliminaries}

As was mentioned before, Wilmshurst's conjecture for $m=1$ can be reformulated in terms of the fixed points. Namely,

Received by the editors May 1, 2001.

2000 Mathematics Subject Classification. Primary 26C10.

The first author was partially supported by an NSF grant DMS-0139008.

The second author was partially supported by an NSF grant DMS-0072312.

(C)2002 American Mathematical Society 
Theorem 1. Let $p(z), \operatorname{deg} p=n>1$, be an analytic polynomial. Then

$$
\#\{z \in \mathbf{C}: \overline{p(z)}=z\} \leq 3 n-2 .
$$

It is easy to see that the fixed points of $\overline{p(z)}$ are finitely many, since the latter are also fixed points of the function $Q(z)=\overline{p(\overline{p(z)})}$ which is an analytic polynomial of degree $n^{2}$. In fact, one can also observe (see 7] and 4]) that for any harmonic function $h(z)=p(z)-\overline{q(z)}, 0<\operatorname{deg} q<\operatorname{deg} p$, all the zeros are isolated. On the other hand, examples of quadratic polynomials show that the estimate of Theorem 1 is the best possible.

2.1. Facts from complex dynamics. If $q(z)$ is a polynomial, a fixed point $z_{0} \in$ $\mathbf{C}$ is attractive, repelling or neutral if, respectively, $\left|q^{\prime}\left(z_{0}\right)\right|<1,\left|q^{\prime}\left(z_{0}\right)\right|>1$ or $\left|q^{\prime}\left(z_{0}\right)\right|=1$. A neutral fixed point is rationally neutral if $q^{\prime}\left(z_{0}\right)$ is a root of unity. We shall say that a fixed point $z_{0}$ attracts some point $w \in \mathbf{C}$ provided that the sequence $q^{k}(w)=\underbrace{q \circ \cdots \circ q}_{k}(w)$ converges to $z_{0}$. A point $\zeta$ is called a critical point of $q$ if $q^{\prime}(\zeta)=0$.

Fact 1. If $\operatorname{deg} q>1, z_{0}$ is an attracting or rationally neutral fixed point, then $z_{0}$ attracts some critical point of $q$.

For the proof, see [2], Ch. III, Thms. 2.2 and 2.3.

2.2. The argument principle. A harmonic function $h=f+\bar{g}$, where $f$ and $g$ are analytic functions, is called sense-preserving at $z_{0}$ if the Jacobian $J_{h}(z)=$ $\left|f^{\prime}(z)\right|^{2}-\left|g^{\prime}(z)\right|^{2}>0$ for every $z$ in some punctured neighborhood of $z_{0}$. We also say that $h$ is sense-reversing if $\bar{h}$ is sense-preserving at $z_{0}$. If $h$ is neither sensepreserving nor sense-reversing at $z_{0}$, then $z_{0}$ is called singular and necessarily (but not sufficiently) $J_{h}\left(z_{0}\right)=0$.

Note that for harmonic functions $z-\overline{p(z)}, \operatorname{deg} p>1$, a point $z_{0}$ is sensepreserving, reversing or singular if and only if $\left|p^{\prime}\left(z_{0}\right)\right|$ is less than 1 , greater than 1 or equal to 1 , respectively.

If $\Gamma$ is an oriented closed curve and $F$ does not vanish on $\Gamma$, then the notation $\Delta_{\Gamma} \arg F(z)$ means the increment of the argument of $F(z)$ along $\Gamma$. We will use the following argument principle which is taken from 4 . The referee pointed out that there is a newer and stronger formulation of the principle found in 3 .

Fact 2. Let $h$ be a harmonic function in a finitely-connected domain $\Omega$ with a piecewise smooth boundary $\Gamma$. Assume that $h$ is continuous in $\bar{\Omega}$ and $h \neq 0$ on $\Gamma$. Suppose also that $h$ has no singular zeros in $\Omega$ and let $N$ be the number of zeros of $h$ inside $\Omega$, counted with their orders and the positive sign for sense-preserving zeros and negative for the sense-reversing ones. Then,

$$
\frac{1}{2 \pi} \Delta_{\Gamma} \arg h(z)=N .
$$

We will apply Fact 2 with $\Omega$ equal to the set of sense-preserving points of $h(z)=$ $z-\overline{p(z)}$, and then to the set of sense-reversing points of $h$ intersected with a suffciently large disk $D(0, R)$, chosen so that $\Gamma \subset D(0, R)$, all zeros of $z-\overline{p(z)}$ are in $D(0, R)$ and the argument change of $z-\overline{p(z)}$ along the circle $C(0, R)$ is $-n$. Then $\frac{1}{2 \pi} \Delta_{C(0, R)} \arg h(z)=-n$, where $C\left(z_{0}, R\right)$ denotes the positively oriented circle with the given center and radius. 


\section{NON-REPELLING FIXED POINTS}

We will now prove Theorem 1. Let us start with the following proposition which is of independent interest.

Proposition 1. If $p$ is a polynomial of degree $n>1$, then the set of points for which $z=\overline{p(z)}$ and $\left|p^{\prime}(z)\right| \leq 1$ has cardinality at most $n-1$.

We consider the function $Q(z):=\overline{p(\overline{p(z)})}$ which is an analytic polynomial of degree $n^{2}$. Notice first that if $\left|p^{\prime}\left(z_{0}\right)\right|=1$ and $\overline{p\left(z_{0}\right)}=z_{0}$, then $Q^{\prime}\left(z_{0}\right)=1$. This follows by writing $\overline{p\left(z_{0}+z\right)}=z_{0}+e^{i \theta} \bar{z}+O\left(|z|^{2}\right)$ with $\theta \in \mathbf{R}$ and iterating. Thus, all points mentioned in Proposition 1 are fixed points of $Q$ which are either attracting or rationally neutral. So, each of them attracts a critical point of $Q$ by Fact 1 .

Lemma 1. If $\overline{p\left(z_{0}\right)}=z_{0}$ and $z \in \mathbf{C}$, then $(\bar{p})^{k}(z) \rightarrow z_{0}$ iff $Q^{k}(z) \rightarrow z_{0}$.

Proof. The $\Rightarrow$ implication is obvious. For the opposite one, we observe that $Q^{k}=$ $(\bar{p})^{2 k}$ by definition, and $(\bar{p})^{2 k}(z) \rightarrow z_{0}$ implies $(\bar{p})^{2 k+1}(z) \rightarrow z_{0}$ since $z_{0}$ is a fixed point.

Recall that a grand orbit under a transformation $F$ is an equivalence class of the relation $x \sim y$ iff $F^{p}(x)=F^{r}(y)$ for some $p, q>0$.

Lemma 2. If $Q^{\prime}(c)=0$, then there are at least $n+1$ critical points of $Q$, counted with multiplicities, which all belong to the same grand orbit under $\bar{p}$.

Proof. Note that if $p^{\prime}(\zeta)=0$, then $\zeta$ and all its preimages by $\bar{p}$ are critical points of $Q$. If $\bar{\zeta}$ is not a critical value, that gives $n+1$ distinct critical points of $Q$ counted with multiplicities. If $p\left(\zeta_{1}\right)=\bar{\zeta}$ and $\zeta_{1}$ is a critical point of $p$ with multiplicity $k$, then $\zeta_{1}$ is a critical point of $Q$ with multiplicity at least $2 k+1$. In any case the sum of multiplicities of critical points of $Q$ over the set $\{\zeta\} \cup \bar{p}^{-1}(\{\zeta\})$ is at least $n+1$.

The condition $Q^{\prime}(c)=0$ implies that either $p^{\prime}(c)$ or $p^{\prime}(\bar{p}(c))$ is 0 and Lemma 2 follows from the remark of the previous paragraph applied to either $c$ or $\bar{p}(c)$, respectively.

Lemma 3. If $Q^{\prime}(c)=0, p\left(z_{0}\right)=\overline{z_{0}}$ and $Q^{k}(c) \rightarrow z_{0}$, then there are $n+1$ critical points of $Q$, counted with multiplicities, all attracted to $z_{0}$ under the iteration of $Q$.

Proof. These critical points are obtained from Lemma 2. By Lemma1, $(\bar{p})^{k}(c) \rightarrow$ $z_{0}$, and then the same must occur for every point in its grand orbit.

As already observed, each point $z_{0}$ which satisfies the conditions of Proposition 1 attracts a critical point of $Q$, but then it attracts $n+1$ of them. Clearly, different fixed points attract disjoint sets of critical points. Since the degree of $Q$ is $n^{2}$, the total number of its critical points counted with multiplicities is $n^{2}-1=(n+1)(n-1)$ which proves the claim of Proposition 1

\section{Proof of the main theorem}

For the purpose of this section, we call the polynomial $p$ regular provided that the conditions $\left|p^{\prime}\left(z_{0}\right)\right|=1$ and $\bar{p}\left(z_{0}\right)=z_{0}$ are not satisfied simultaneously for any $z_{0} \in \mathbf{C}$. 
Lemma 4. If $p$ is regular of degree $n>1$, then there are at most $2 n-1$ points $z$ in the complex plane for which both $\bar{p}(z)=z$ and $\left|p^{\prime}(z)\right|>1$ are satisfied.

Proof. Consider the regions $\Omega_{+}$where $z-\bar{p}(z)$ is sense-preserving and $\Omega_{-}$where it is sense-reversing. They are separated by a piecewise oriented analytic curve (a lemniscate) $\Gamma$ which is the boundary of $\Omega_{+}$. In addition, make $\Omega_{-}^{0}$ compact by intersecting $\Omega_{-}$with a large disk $D(0, R)$ chosen so that $\Gamma \subset D(0, R)$, all zeros of $z-\overline{p(z)}$ are in $D(0, R)$ and the argument change of $z-\overline{p(z)}$ along the circle $C(0, R)$ is $-n$. By Fact 2 and Proposition $1, \Delta_{\Gamma}(z-\overline{p(z)}) \leq 2 \pi(n-1)$. Hence,

$$
\Delta_{C(0, R)}-\Delta_{\Gamma} \geq-2 \pi(2 n-1) .
$$

Since $C(0, R)-\Gamma$ is the oriented boundary of the region $\Omega_{-}^{0}$, Fact 2 means that $-\frac{1}{2 \pi}\left(\Delta_{C(0, R)}-\Delta_{\Gamma}\right)$ is the number of zeros of $z-\overline{p(z)}$ in $\Omega_{-}$, which is what the lemma claims.

From Proposition 1 and Lemma 4 we see that Theorem 1 holds for regular $p$. Moreover, it also holds on the closure of the set of regular polynomials (with the topology of uniform convergence in the spherical metric). Indeed, a sufficiently small perturbation will not decrease the number of zeros of $z-\bar{p}(z)$ in $\Omega_{-}$, hence Lemma 4 still holds for $p$ in the closure of the set of regular polynomials.

It remains to see that the set of regular polynomials is dense and we show even more:

Lemma 5. If $p(z)$ is a polynomial of degree greater than 1 , then the set of complex numbers $c$ for which $p(z)-c$ is regular is open and dense in $\mathbf{C}$.

Proof. This lemma may be derived from general considerations about algebraic sets. Here we give a simple proof due to D. Sarason.

For a given $p$, consider the set $S$ which is the image under the transformation $z \rightarrow p(z)-\bar{z}$ of the set $\left\{z \in \mathbf{C}:\left|p^{\prime}(z)\right|=1\right\}$. If $c \notin S$, then $p(z)-c-\bar{z} \neq 0$ whenever $\left|p^{\prime}(z)\right|=1$, in other words $p(z)-c$ is a regular polynomial. But $S$ is

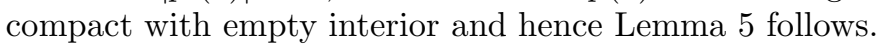

Theorem 1 is now proved.

\section{FinAl REMARKS}

Sharpness of the result. As was mentioned before, easy examples of polynomials of degree 2 and 3 show that when considered for all $n>1$, the estimate of Theorem 1 is sharp. For example, the equation

$$
\frac{1}{2}\left(z^{3}-3 z\right)+\bar{z}=0
$$

has seven roots: $0, \pm 1, \frac{1}{2}( \pm \sqrt{7} \pm i)$, with any combinations of the signs in the last pattern allowed. This realizes the bound $3 n-2$. Moreover, there are two roots \pm 1 at which the function is sense-preserving, and five sense-reversing roots realizing the estimates of Proposition 10 and Lemma 4

However, if Theorem 1 is sharp for every $n \geq 2$ remains to be seen. Along those lines B. Crofoot and D. Sarason [5] raised the following important question.

Conjecture 1. For $n>1$ there exist $n-1$ points $z_{1}, \ldots, z_{n-1}$ and a polynomial $p$ of degree $n$ such that $p\left(z_{j}\right)=\overline{z_{j}}$ and $p^{\prime}\left(z_{j}\right)=0$ for all $j$. 
If true, this implies that the bound of Proposition 1 is sharp for each $n$, and then so are the bounds of Lemma 4 and Theorem 1

On Proposition 1, An analogue of that proposition with $p(z)$ replacing $\overline{p(z)}$ has a cute elementary proof which does not use Fact 1 and is sketched below.

Proposition 2. Let $p(z)$ be a polynomial of degree $n>1$. Then, the number of its fixed points with derivative in the set $\overline{D(0,1)} \backslash\{1\}$ is at most $n-1$.

Let $a_{1}, \ldots, a_{n}$ be the fixed points of $p$. Then

$$
p(z)=z+C\left(z-a_{1}\right) \cdots\left(z-a_{n}\right)=: z+q(z)
$$

with $C \in \mathbf{C}, C \neq 0$. If $p^{\prime}\left(a_{j}\right)=1$ for some $j$, then the claim is obvious, so without loss of generality all $a_{j}$ are simple fixed points. To see that $\left|p^{\prime}\left(a_{j}\right)\right|>1$ for some $j$, it suffices to show that the points $q^{\prime}\left(a_{j}\right)$ cannot all belong to the closed unit disk centered at -1 with 0 excepted. To this end, we demonstrate that 0 belongs to the convex hull of points $q^{\prime}\left(a_{j}\right), j=1, \ldots, n$. This follows at once since

$$
\sum_{j=1}^{n} \frac{1}{q^{\prime}\left(a_{j}\right)}=C_{1} \sum_{j=1}^{n} \operatorname{res} \frac{1}{q(z)}=0
$$

where

$$
\frac{1}{q^{\prime}\left(a_{j}\right)}=\frac{\overline{q^{\prime}\left(a_{j}\right)}}{\left|q^{\prime}\left(a_{j}\right)\right|^{2}}
$$

and so equality (1) indeed means that 0 can be realized as a convex combination of $q^{\prime}\left(a_{j}\right)$.

Examples of the form

$$
p(z)=(1+\epsilon) z+z^{n}, \quad n \geq 2,0<\epsilon<\frac{2}{n-1},
$$

show that the bound of Proposition 2 is the best possible for any $n$.

Possible extensions. Wilmshurst has conjectured (see 7]) that for a general $h=p(z)-\overline{q(z)}, \operatorname{deg} p=n>m=\operatorname{deg} q>0$, the maximal number of zeros is $m(m-1)+3 n-2$. It is not clear whether the ideas of this paper can be extended sufficiently to treat his conjecture. Even in the simplest case $q(z)=z^{m}$, $1 \leq m \leq n-1$, it immediately requires a profound study of the dynamics of the

map $\sqrt[m]{\overline{p(z)}}$ on the Riemann surface. Perhaps, such an investigation will lead to a beginning of a new tale.

\section{REFERENCES}

[1] D. Bshouty, W. Hengartner \& T. Suez: The exact bound of the number of zeros of harmonic polynomials, J. d'Analyse Math. 67 (1995), 207-218 MR 97f:30025

[2] L. Carleson \& T. Gamelin: Complex Dynamics, Springer-Verlag, New York-Berlin-Heidelberg (1993) MR 94h:30033

[3] M. Cristea: A generalization of the argument principle, Compl. Var. Theory Appl. 42 (2000), 335-345 MR 2001d:30085

[4] P. Duren, W. Hengartner \& R.S. Langesen: The argument principle for harmonic functions, Amer. Math. Monthly 103 (1996), 411-415 MR 97f:30002

[5] D. Sarason, written communication, Feb. 1999, Oct. 2000 
[6] T. Sheil-Small in Tagesbericht, Mathematisches Forsch. Inst. Oberwolfach, Funktionentheorie, 16-22.2.1992, 19

[7] A.S. Wilmshurst, The valence of harmonic polynomials, Proc. AMS 126 (1998), 2077-2081 MR 98h:30029

Department of Mathematics, University of Arkansas, Fayetteville, Arkansas 72701

E-mail address: dmitry@comp.uark.edu

Department of Mathematics, Pennsylvania State University, University Park, PennSYLVANIA 16802

E-mail address: swiatek@math.psu.edu 